\title{
La formación inicial docente en Chile: Una revisión bibliográfica sobre su implementación y logros
}

\author{
Initial Teaching Training in Chile: A Bibliographic Review on Its Implementation and \\ Achievements
}

\section{A formação inicial docente no Chile: uma revisão bibliográfica sobre sua implementação e conquistas}

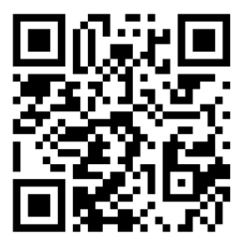

Lady Sthefany Bastías-Bastías

Universidad de Talca

Facultad de Psicología

Talca, Chile

lady.bastias@utalca.cl

https://orcid.org/0000-0002-6228-1894

Carolina Iturra-Herrera

Universidad de Talca

Facultad de Psicología

Talca, Chile

citurra@utalca.cl

https://orcid.org/0000-0003-2094-1302

Recibido • Received • Recebido: 12 / 01 / 2020

Corregido • Revised • Revisado: 10 / 08 / 2021

Aceptado • Accepted • Aprovado: 09 / 10 / 2021

\begin{abstract}
Resumen:
Introducción. En Chile, durante las últimas décadas se han implementado diversas políticas educativas orientadas a mejorar la formación inicial docente, con el propósito de impactar en la calidad del sistema educativo. Han sido varias las investigaciones que han descrito el resultado en la implementación de tales políticas. Objetivo. Se espera describir los hitos más importantes para la formación inicial del profesorado en Chile y analizar, con base en las investigaciones, los resultados de estas políticas. Análisis. Se efectúa una búsqueda de textos académicos publicados entre los años 2010 y 2019 presentes en las bases de datos Google Scholar, Web of Science y Scopus que contengan los conceptos: initial teacher training, educational policy y Chile. Resultados. Las políticas promovidas por el Estado han sido implementadas por las universidades que forman docentes. Asimismo, los resultados de la Prueba inicia, que evalúa los conocimientos disciplinarios y pedagógicos de estudiantes de la carrera de pedagogía que se encuentran en el último año de formación, demuestra que el profesorado no alcanza los estándares de desempeño esperados, cuyas debilidades son percibidas por las personas egresadas de las carreras. Conclusiones. Las políticas implementadas no lograron mantener un alza en el acceso a las carreras de pedagogía. Tampoco acortaron la distancia entre la formación universitaria
\end{abstract}


http://doi.org/10.15359/ree.26-1.13

http://www.una.ac.cr/educare

educare@una.ac.cr

con la formación práctica en los establecimientos educacionales, y existe una débil correlación entre los mecanismos de control instaurados. Como recomendaciones se propone ampliar los criterios de búsqueda integrando la política de gratuidad y década de los años noventa.

Palabras claves: Enseñanza superior; formación de docentes; política educacional; formación inicial; Chile.

\begin{abstract}
:
Introduction. In Chile, several educational reforms and policies aimed at improving initial teacher training have been generated and implemented in recent decades to impact the quality of the education system. In this context, several studies ought to describe the outcome of implementing such reforms and policies. Aim. To describe the central milestones in initial teacher training in Chile, analyzing them in the context of initial teacher training policies in the country. Method. A scientific literature review was conducted for academic texts published between 2010 and 2019, using the Google Scholar, Web of Science, and Scopus databases containing the concepts initial teacher training, educational policy, and Chile. Results. The results indicate that the policies promoted by the State have been implemented by the universities that train teachers. In addition, the results of the Inicia Test (which assesses professional knowledge and skills of final year Education students) demonstrate that teachers do not reach the expected disciplinary and pedagogical performance standards weaknesses perceived by career graduates. Conclusions. Initial teacher training policies could not steadily increase the number of students interested in Education training, nor could they reduce the gap between university training and actual professional practice. There is a weak correlation among the mechanisms of control implemented. As a recommendation, we suggest broadening the scope of the search so as to include government financial policy on higher education since the 1990s.
\end{abstract}

Keywords: Higher education; teacher training; educational policy; initial training; Chile.

\title{
Resumo:
}

Introdução. No Chile, nas últimas décadas foram geradas e implementadas diversas políticas educativas orientadas a melhorar a formação inicial docente, com o propósito de impactar na qualidade do sistema educativo. Várias pesquisas têm procurado descrever a implementação e o resultado dessas políticas. Objetivo. Descrever os marcos centrais da formação inicial de professores no Chile, analisando-os no contexto das políticas de formação inicial de professores no país. Metodologia. Para isso, foi realizada uma busca de textos acadêmicos publicados entre os anos de 2010 e 2019 que constam nas bases de dados Google Scholar, Web of Science e Scopus. Os seguintes conceitos foram analisados: initial teacher training, educational policy e Chile. Resultados. As políticas promovidas pelo Estado foram implementadas pelas universidades que treinam professores. Da mesma forma, os resultados da prova inicial, que avalia os conhecimentos didáticos e pedagógicos de estudantes da carreira de pedagogia demonstram que os professores não atendem aos padrões esperados de desempenho, cujas fraquezas são percebidas pelos formandos das carreiras. Conclusão. As políticas implementadas não lograram manter um aumento no acesso as carreiras de pedagogia. Nem diminuíram a distância entre a formação universitária com a formação prática nos estabelecimentos educativos, existindo uma fraca correlação entre os mecanismos de controle implementados. Como recomendação, sugere-se ampliar a perspectiva da pesquisa, de forma a incluir a política de gratuidade e da década de 90.

Palavras chaves: Ensino superior; formação de docentes; política educacional; educação inicial; Chile. 
http://doi.org/10.15359/ree.26-1.13

\section{Introducción}

La primera entidad responsable de formar el profesorado en Chile nace el año 1842 con la creación de la Escuela Normal de Preceptores que prepararía a los futuros grupos de docentes de educación primaria. Por su parte, el Instituto Pedagógico de la Universidad de Chile sería la entidad encargada de formar a docentes de secundaria durante el siglo XIX. En efecto, podemos ver que tempranamente se realiza una distinción de los organismos estatales encargados de formar a docentes de enseñanza primaria y secundaria, condicionada en el caso de las escuelas normales por su origen social, y funcionalidad a la sociedad (Cox y Gysling, 2009). No obstante, ambas entidades ofrecían una incipiente trayectoria formativa que comenzaba con la selección del futuro profesorado, un compromiso laboral y un régimen de internado. A partir de los años sesenta, la educación normal se asimila con la formación secundaria, y vive una cierta estabilización con reformas que regulan la gratuidad y funcionamiento de la educación chilena desde 1920 con la Ley de Instrucción Primaria.

A mediados de los años setenta, la formación de docentes y el sistema educacional chileno comienzan a experimentar reformas sustanciales que acaban con la formación de profesorado normalista y se inicia el traspaso del sistema educativo hasta ese entonces estatal, a las municipalidades. Como consecuencia de estas reformas impuestas durante el gobierno autoritario que tuvo Chile desde 1973 al 1990, es posible mencionar la baja sostenida en la matrícula en las carreras de pedagogía, el deterioro de la condición profesional del personal docente chileno rebajado a profesional no-universitario; y el énfasis en la formación disciplinar técnica en desmedro de la formación con los ideales de ilustración que fueron el motor de la formación de profesorado secundario (Ávalos, 2014; Fardella y Sisto, 2015; Núñez Prieto, 2007).

Con el retorno a la democracia en Chile, a partir de los años noventa, se impulsan una serie de programas tendientes a mejorar el sistema escolar. Varias de las acciones estarán centradas en mejorar la calidad de la enseñanza, lo que llevará a impulsar propuestas para fortalecer la formación del profesorado. Ejemplo de ello fue la inscripción a programas internacionales como el proyecto Tuning Latinoamérica, la creación del programa de Fortalecimiento a la Formación Inicial Docente (FFID); y el Programa de Mejoramiento de la Calidad y Equidad de la Educación Superior (MECESUP), entre otros. Estos programas vinieron a hacerse cargo de la desregulación que sufrió la formación del profesorado chileno, la que trajo como consecuencia que las carreras de pedagogía comenzaran a transitar bajo lógicas del mercado, ofertándose en universidades públicas y privadas sin mayor regulación. A ello se suma la dudosa calidad de las instituciones de educación superior que ofrecían las carreras de pedagogía, cuyos procesos de acreditación rondaban entre los tres o menos años y "solo un siete por ciento tiene seis o más años de acreditación" (Panel de Expertos para una Educación de Calidad [PEEC], 2010, p. 22).

Ahora bien, en los últimos cinco años, las universidades que forman a las futuras generaciones de docentes vuelven a ser parte de las exigencias que ha impulsado el Estado 
http://doi.org/10.15359/ree.26-1.13

http://www.una.ac.cr/educare

educare@una.ac.cr

chileno para regular la formación inicial del profesorado. Un ejemplo actual es la puesta en marcha de la ley que crea el Sistema de Desarrollo profesional docente, publicada el 1 de abril del año 2016 (Ministerio de Educación [MINEDUC], 2016).

Si bien la formación de profesorado siempre ha sido objeto de reformas, esta última, en particular, sienta las bases de cambios sustanciales de cómo se ha llevado a cabo la formación docente en los últimos treinta años y retoma ciertos desafíos pendientes de las reformas implementas en los años noventa. Así, estos antecedentes revelan la importancia que tiene este estudio como estado del arte de los principales programas que han sido instaurados en la formación de profesorado chileno, y pone en tensión los resultados de estos programas a la luz de la evidencia empírica recolectada y la ejecución de esos mismos tras diez años de implementación de las política o programas. Abordar la temática de la preparación de docentes es fundamental para asegurar la calidad de los sistemas educativos (Vaillant, 2018) y conlleva siempre desafíos para las políticas educativas. Por lo tanto, el objetivo que guiará esta revisión bibliográfica es describir los hitos más importantes para la formación inicial de profesorado en Chile y analizar con base a las investigaciones los resultados de estas políticas.

Para dar respuesta a este objetivo se analizarán dieciocho artículos sobre la formación inicial del profesorado chileno. De su análisis emergen cinco categorías que permiten dar respuesta al objetivo del estudio: 1) Regulaciones a la formación del profesorado en Chile; 2) Caracterización de estudiantes que ingresan a estudiar pedagogía; 3) Propuestas a la política para la formación inicial docente; 4) Debilidades y aportes para los programas de formación inicial; y 5) Estándares y resultados de la Prueba Inicia.

\section{Método}

La estrategia utilizada para la revisión bibliográfica incluyó un estudio de los documentos presentes en las bases de datos de Google Scholar, Scopus y Web of Science. Se utilizaron dos criterios de selección para la búsqueda de la información. El primer criterio de carácter temporal, donde se realizó una búsqueda de artículos especializados de los últimos nueve años (2010-2019). El otro criterio de búsqueda fue de carácter conceptual en tres metabuscadores. En el caso del buscador Google scholar se usaron los descriptores: formación inicial docente y Chile solo en el título. Mientras que en el buscador Scopus y Web of Science, se utilizaron los descriptores: initial training teacher, education policy y Chile que estuvieran presentes a la vez en el título, abstract y palabras claves. Como criterio de inclusión se utilizaron artículos, capítulos de libros o libros completos, indexados; artículos de acceso abierto, el tramo temporal definido para la búsqueda y temáticas que abordaran la educación superior chilena. A continuación, se presenta un resumen de los criterios de inclusión y exclusión para el proceso de búsqueda de la información (ver Tabla 1). 
http://doi.org/10.15359/ree.26-1.13

Tabla 1: Criterios de inclusión y exclusión para la selección de artículos

- Artículos indexados en Scopus, Web of Science o Google scholar

- Ponencias, publicaciones en periódicos, tesis,

- Artículos en idioma español e inglés testimonios, reseñas, entrevistas.

- Artículos Open Access

- Referencia a otros países de Latinoamérica distintos de Chile

- Artículos publicados desde el 2010 al 2019

- Estudio de caso de formación disciplinar de una universidad en particular

- Artículos sobre educación superior chilena

Nota: Elaboración propia.

Los referentes conceptuales utilizados para esta revisión, ya sea formación inicial y política educativa, son dos términos que se encuentran relacionados y que permiten explicar los hitos por los que ha transitado la formación inicial del profesorado chileno. Sobre el concepto de formación inicial, es entendido como un proceso de apropiación de los saberes en una institución que forma para aquella área (Gysling, 1992, en Sánchez, 2014). En el caso de esta revisión se asume que es el momento a través del cual el futuro profesorado recorre una trayectoria en la que adquiere diversos saberes. Por su parte, el concepto de política educativa es el eje que contiene las reformas que se desarrollan en los sistemas educativos, donde se realizan "transformaciones democrático-modernizadoras en la estructura del sistema y las prácticas educativas" (Núñez, 1997, en Oliva, 2010, p. 311). Cabe señalar que la caracterización definitiva del corpus que compone esta revisión corresponde a 18 documentos publicados desde el año 2010 hasta el año 2019, indexados y de acceso abierto.

\section{Resultados}

Como se mencionó anteriormente, del análisis de los artículos presentes en este estudio emergieron cinco categorías: 1) Regulaciones a la formación de profesorado en Chile; 2) Caracterización de estudiantes que ingresan a estudiar pedagogía; 3) Propuestas a la política para la formación inicial docente; 4) Debilidades y aportes para los programas de formación inicial; y 5) Estándares y resultados de la "Prueba Inicia”. Estas categorías dan cuenta de los temas destacados por las autorías en torno a los programas, así como también evalúan el impacto de las políticas implementadas en la formación de docentes, las que a continuación se detallan. 
http://doi.org/10.15359/ree.26-1.13

http://www.una.ac.cr/educare

educare@una.ac.cr

\section{Regulaciones a la formación del profesorado en Chile}

Los estudios coinciden en mencionar al menos cuatro programas o políticas que han propiciado un mejoramiento sostenido hacia la formación de profesorado en Chile. El primero es el programa de Fortalecimiento a la Formación Inicial Docente (FFID); posteriormente la creación del Programa de Mejoramiento de la Calidad y Equidad de la Educación Superior (MECESUP); el rol del Consejo Nacional de Acreditación de pregrado (actual CNA); y finalmente, el diseño y aplicación en las facultades de educación del Programa Inicia.

El programa de Fortalecimiento a la Formación Inicial Docente (FFID) se sitúa en esta revisión como uno de los pilares que estructuran las mejoras respecto a la cuestión docente (Bellei, 2010) implementados por los gobiernos de la Concertación. Su anuncio se realiza en el contexto de la cuenta pública del entonces presidente de la república Eduardo Frei Ruiz-Tagle. Durante ese discurso, se señala que se priorizarán las reformas a la educación (Castro Rubilar, 2017). Este programa tuvo por objetivo "estimular procesos de cambio en una formación docente muy debilitada durante los años de dictadura, tanto en sus recursos académicos como en el número reducido y baja calidad de los interesados en ingresar a las carreras de pedagogía" (Ávalos, 2014, p. 15).

Algunos textos (Contreras-Sanzana y Villalobos-Clavería, 2010; Ferrada et al., 2013) coinciden en que la implementación del programa FFID que comenzó en el año 1997 y terminó el 2002, generó tres consecuencias para las 17 universidades que participaban. La primera de ellas: repercutió en la actualización de los programas de estas universidades que abarcaban aproximadamente el $80 \%$ de las matrículas de estudiantes de las carreras de pedagogía (Cox et al., 2010; Ferrada et al., 2013; Rodríguez Garcés y Castillo Riquelme, 2014; Soto-Hernández y Díaz Larenas, 2018). La segunda consecuencia también en el ámbito curricular: acusó la escasa articulación existente entre la formación pedagógica y la formación práctica recibida en los años de formación, que, hasta entonces, estaba solo presente el último año de formación. Ese diseño formativo propiciaba aún más la desconexión entre formación inicial y la realidad escolar de los establecimientos educacionales chilenos (Cox et al., 2010; Ferrada et al., 2018; Pavié Nova, 2012). Finalmente: la implementación de este programa significó para las universidades, robustecer sus claustros académicos, dotándolos de desarrollo profesional, lo que produjo un "aumentando el grado de magíster de 27 a 45\% y el grado de doctor de 13 a 23\%" (ContrerasSanzana y Villalobos-Clavería, 2010, p. 410).

La segunda de las medidas implementadas desde el año 2000 hasta la actualidad es el establecimiento del Programa de Mejoramiento de la Calidad y Equidad de la Educación Superior (MECESUP). Este programa fue un convenio entre el Banco Mundial y el Estado chileno para impulsar acciones de progreso en las instituciones de educación superior en sintonía con los lineamientos del Programa Tuning para Latinoamérica, lo que además conllevaría procesos de accountability (Reich, 2009). En un primer momento, desde el año 1999 al 2004, los MECESUP 
http://doi.org/10.15359/ree.26-1.13

estuvieron destinados especialmente al mejoramiento de la infraestructura universitaria, y la adquisición de recursos de apoyo para la enseñanza en las universidades pertenecientes al Consejo de Rectores de Chile (CRUCH) (Ferrada et al., 2013; MINEDUC, 2019; Reich, 2009). Posteriormente el MECESUP-2 (2006 al 2008) tuvo, entre sus objetivos, instaurar innovaciones académicas en las carreras de pedagogías (Cox et al., 2010). Quizás uno de los cambios más importantes para la pedagogía fue el ajuste de los programas de formación al modelo basado en competencias y el establecimiento del sistema de créditos transferibles (SCT-chile) que, en el ideal, permitiría la transferencia y movilidad estudiantil dentro de Chile como en Latinoamérica (Ferrada et al., 2013; Gaete et al., 2016; Ruffinelli, 2013). En la actualidad este programa continúa vigente con el objetivo de ayudar a las instituciones de educación terciaria a alcanzar la excelencia (MINEDUC, 2019).

La creación del Sistema de Acreditación de carreras de Pregrado (actual Comisión Nacional de Acreditación, CNA) surge el año 2004 y tiene por objetivo verificar y promover la calidad de la educación en Chile para las carreras de pedagogía (Ávalos, 2014; Cox et al., 2010). No obstante, a partir del año 2006, con la creación de la Ley de Aseguramiento de la Calidad de la Educación Superior (Ley 20.129) este mecanismo tuvo entre sus funciones acreditar, de forma obligatoria, las instituciones, así como también los programas que formaban docentes. Esto significó comenzar a evaluar la calidad de la formación inicial entregada por las universidades de acuerdo con el modelo basado en competencias o resultados de aprendizaje (Ferrada et al., 2013; Gaete et al., 2016; Ruffinelli, 2013; Soto-Hernández y Díaz Larenas, 2018). Finalmente, por medio de agencias acreditadoras privadas -cuya figura se elimina el año 2017-, se comienza a velar por la coherencia de los proyectos formativos en las carreras de pedagogía (Cox et al., 2010; Ferrada et al., 2018).

Por último, el Programa Inicia es uno de las políticas más recientes. Fue creado el año 2008 y poseía tres componentes: El primero es "la definición de estándares y orientaciones curriculares para cada carrera de Pedagogía, con el fin de otorgar una base de conocimientos comunes acordes a los requerimientos del sistema educativo" (Alvarado et al., 2010, p. 25). El segundo componente de esta política, implicaba la instalación de un programa de apoyo a las universidades para la innovación curricular (Cox et al., 2010; Ferrada et al., 2013; Gaete et al., 2016; Rodríguez Garcés y Castillo Riquelme, 2014); mientras que el tercer componente de este programa, involucraba el diseño y aplicación de una evaluación al final de la carrera, llamada Prueba Inicia. Esta evaluación tenía por objetivos evaluar los desempeños alcanzados por los estudiantes de pedagogía en el último año de su formación (Ávalos, 2014; Gaete et al., 2016; Ruffinelli, 2013); proporcionar información del nivel de logro alcanzado por las personas egresadas en ámbitos pedagógicos y disciplinares; y tomar decisiones con base en los resultados que permitan diseñar políticas de Estado para mejorar la formación docente (Cox et al., 2010; Pedraja-Rejas et al., 2012; Soto-Hernández y Díaz Larenas, 2018). 
http://doi.org/10.15359/ree.26-1.13

http://www.una.ac.cr/educare

educare@una.ac.cr

Respecto de esta última medida, la actual Ley 20.903 que crea el Sistema de Desarrollo Profesional Docente ha robustecido las indicaciones propuestas en el Programa Inicia. En la actualidad establece puntajes para el ingreso a las pedagogías, mantiene los sistemas de acreditación y reemplaza la ex Prueba Inicia por la Evaluación Nacional Diagnóstica (END) (MINEDUC, 2016). La END permite conocer los desempeños alcanzados por las futuras personas docentes; pero, al contrario de su antecesora, se aplica un año antes de que el estudiantado egrese. Esta medida tiene por objetivo que las universidades establezcan mejoras en aquellos aprendizajes que no demostraron un buen desempeño (Centro de Perfeccionamiento, Experimentación e Investigaciones Pedagógicas [CPEIP], 2019).

Las medidas instauradas para el fortalecimiento de la profesión docente en Chile son vistas positivamente por los estudios. No obstante, se observa con cautela la instauración cada vez más recurrente de procesos de accountability en la educación superior y en particular en la formación de profesorado (Montecinos, 2014). Así, entre los artículos que analizan las propuestas de reformas a la FID en Chile, se indica que estas no hacen más que instaurar un fuerte control sobre las facultades de educación a través de la creación de "un régimen de números derivados de exámenes y acreditaciones" (Ball, 2010 en Montecinos, 2014, p. 287).

\section{Caracterización de estudiantes que ingresan a estudiar pedagogía}

El mecanismo de selección para ingresar a una carrera universitaria en Chile era a través de la Prueba de Aptitud Académica (PAA), la que, desde el año 2003, fue reemplazada por la Prueba de Selección Universitaria (PSU) (Pavié Nova, 2012). Por medio de este mecanismo, la mayor parte de estudiantes que ingresan a estudiar carreras de pedagogía superan escasamente el puntaje mínimo requerido por las universidades públicas (Ávalos, 2004). Es decir, las carreras que forman profesorado chileno no están consideradas como una opción conveniente para estudiantes con elevados puntajes en las pruebas de ingreso a la universidad, quienes optan mayoritariamente por carreras denominadas tradicionales (ingenierías, medicina, derecho, etc.). A su vez, el descendido puntaje de ingreso para estudiar carreras de pedagogía también está asociado a un nivel socioeconómico bajo que sitúa, a estudiantes que se matriculan, en los dos primeros quintiles de menores ingresos de la población chilena (Inzunza et al., 2011; Pavié Nova, 2012). No obstante, estudiar una carrera universitaria como educación, pese a que posee escasa selectividad, significa para este segmento de la población, la posibilidad certera y estable de acceder a una movilidad social (Cox et al., 2010).

Otro de los fenómenos que a la luz de los autores permitiría caracterizar quiénes son los estudiantes que ingresan a pedagogía, se explica por la dependencia universitaria en las que se matriculan. Insunza etal.(2011) afirman queel estudiantado matriculado durante el 2008 en alguna de las carreras de pedagogía corresponde a ciento tres mil estudiantes, donde un $73,6 \%$ se concentraba en instituciones de educación superior privada en la región metropolitana. Esta tendencia se replica 
http://doi.org/10.15359/ree.26-1.13

en la distribución nacional donde estudiantes acceden en un 68,4\% a instituciones de educación superior de carácter privada. Por su parte, se señala que en el año 2008 y solo en el caso de la carrera de educación básica, el $84 \%$ de la matrícula se concentraba en instituciones de educación superior con escasa o nula selectividad. Esta situación que significa una alta demanda hacia este tipo de universidades responde a que el puntaje de ingreso para estudiar pedagogía era obligatorio solo para las universidades estatales chilenas (Ávalos, 2004; Cox et al., 2010).

Junto con las regulaciones en términos de políticas y programas que se instalaron para fortalecer la formación inicial de docentes (1998-2002), se establecen múltiples incentivos. Entre ellos se mencionan becas dirigidas a estudiantes con notas de excelencia y buenos resultados en la PAA, estudiantes que tuvieran experiencia en tareas pedagógicas sin estudios profesionales y con licenciatura de otras disciplinas afines a la educación (Ávalos, 2004; Bellei, 2010; Gaete et al., 2016). Posteriormente desde el año 2010, se crea la beca Vocación de profesor, que iba dirigida a estudiantes que tuvieran más de 600 puntos en la PSU y que quisieran estudiar una carrera de pedagogía en una universidad estatal acreditada por al menos dos años (Rodríguez Garcés y Castillo Riquelme, 2014). Atraer a estudiantes de mayores puntajes para estudiar la carrera de pedagogía fue uno de los temas centrales de algunas políticas descritas para mejorar la formación inicial.

La Beca Vocación de Profesor provocó un alza en los puntajes alcanzados en el año 2011, donde el $68 \%$ del futuro profesorado contaba con puntajes sobre 600 puntos en la PSU yestudiantes que poseían sobre 700 puntos PSU se triplicaron (Rodríguez Garcés y Castillo Riquelme, 2014). Sin embargo, también provocó efectos no esperados que acrecentaron las brechas en la cantidad de matrícula en universidad con escasa selectividad y puntajes más bajos en detrimento de las Universidades del Consejo de Rectores (CRUCH) que vieron sus matrículas descendidas dada las condiciones de selectividad que debían tener para implementar la beca Vocación de profesor (Cox et al., 2010; Rodríguez Garcés y Castillo Riquelme, 2014; Sotomayor y Gysling, 2011).

\section{Propuestas a la política para la FID, desafíos y temas pendientes}

El establecimiento de políticas como las descritas en el apartado anterior, han contribuido a regular la condición de la formación de docentes en Chile. No obstante, es necesario mirar la implementación y los resultados de estas mismas. Por consiguiente, entre los artículos revisados, cuatro de ellos realizan propuestas de mejora a las políticas implementadas al considerar revisiones teóricas y experiencias internacionales.

Una de las recomendaciones establecidas por el Panel de Expertos para una Educación de Calidad (PEEC) fue la incorporación paulatina de la alianza entre las universidades y los establecimientos educacionales, lo que se denominó en el informe como "redes de escuelas, liceos y colegios como 'campos de práctica profesional'"' (Alvarado et al, 2010, p. 7). Esta asociación posibilitaría el fortalecimiento de la formación práctica que realizan estudiantes 
http://doi.org/10.15359/ree.26-1.13

http://www.una.ac.cr/educare

educare@una.ac.cr

de pedagogía durante su trayectoria formativa. Asimismo, los diagnósticos elaborados por agencias educativas internaciones refuerzan la escasa existencia de la inmersión temprana del futuro estudiantado a las escuelas y la desconexión con los programas formativos (Organización para la Cooperación y el Desarrollo Económicos [OCDE], 2010).

En este sentido, Gaete el al. (2016) advierten que aún existe consenso entre grupos investigadores y personal experto sobre esta brecha, que pese a las reformas instauradas aún persiste. Se indica, también, que la relación entre formación inicial y establecimientos escolares es uno de los puntos relevantes a abordar en los procesos de innovación curricular de las carreras de pedagogía y así evitar la desconexión entre la formación universitaria y el quehacer profesional (Gaete et al., 2016; Montecinos, 2014). De este modo, la asociación entre ambas instituciones podría propiciar una especie de trayectoria profesional donde se generen aprendizajes desde las primeras inmersiones en el contexto escolar (Contreras-Sanzana y Villalobos-Clavería, 2010; Gaete et al., 2016) y posibilitaría que el personal docente destacado de los establecimientos educacionales nutriera los programas formativos de las universidades (Montecinos, 2014). Por otra parte, el rol del personal docente en ejercicio es relevado, según los estudios, como actores claves conocedores del ejercicio de la docencia y también como posibles articuladores entre el acompañamiento de profesorado nóvel en su inserción en las aulas escolares (Contreras-Sanzana y Villalobos-Clavería, 2010; Gaete et al., 2016; Montecinos, 2014).

\section{Debilidades y aportes para los programas de formación inicial}

Entrelas consecuencias dela escasa conexiónqueexisteentrelaformación inicialyel ejercicio profesional del personal docente, cinco investigaciones han analizado desde la formación en servicio, las debilidades de la formación recibida por estudiantes que egresaron entre el 2008 y el 2011 (Ferrada et al., 2013, 2018; Gaete et al., 2016; Ruffinelli, 2013; Soto-Hernández y Díaz Larenas, 2018); mientras que otras dos investigaciones dan cuenta de las percepciones de estas políticas educativas desde la mirada del personal director de los establecimientos chilenos (Weinstein et al., 2018; Weinstein et al., 2019). A continuación, se describirán los objetivos, metodologías y principales resultados de cada uno de los artículos de investigaciones.

Uno de los estudios desarrollados por Ruffinelli (2013) tuvo por objetivo conocer la percepción de la formación recibida por 890 estudiantes que egresaron de la carrera de pedagogía entre los años 2008 al 2011, y que tenían al menos tres años de ejercicio profesional. La metodología utilizada fue un estudio comparativo de tipo cuantitativo, a través de la aplicación de una encuesta online a estudiantado egresado de las carreras de pedagogía básica de distintas IES. Los resultados de esta investigación reflejan que existen distintas debilidades percibidas por el estudiantado egresado de universidades selectivas respecto de aquel estudiantado que egresó de universidades con escasa o nula selección. Las debilidades señaladas por estudiantes de IES selectivas son: 
http://doi.org/10.15359/ree.26-1.13

http://www.una.ac.cr/educare educare@una.ac.cr

- la formación para el trabajo con padres y familias,

- la formación para el manejo de sus estudiantes: para el manejo del comportamiento, de la diversidad y del clima de aula; y

- debilidades en la formación disciplinar en ciencias naturales (Ruffinelli, 2013, p. 148).

En tanto, las IES con nula o baja selectividad indican tener debilidades en la formación didáctica de las asignaturas de lenguaje y matemática, y escasa formación sobre el sistema educacional chileno (Gaete et al., 2016; Ruffinelli, 2013).

Las debilidades percibidas por el personal docente son solo el inicio de otras debilidades producidas durante la trayectoria formativa. Si se analizan los resultados de los aprendizajes que lideran estas personas docentes en las evaluaciones estandarizadas como SIMCE o la Prueba Inicia, es posible establecer que los resultados en estas mediciones distan de la buena percepción que indican tener respecto a su formación inicial en las áreas pedagógicas y disciplinares (CPEIP, 2019; MINEDUC, 2019; Ruffinelli, 2013). Esta evidencia podría ser reflejo del análisis superficial y poco crítico que realiza el personal docente nóvel sobre su proceso de formación (Ferrada et al., 2013; Ruffinelli, 2013).

Soto-Hernández y Díaz Larenas (2018) analizan la percepción de personas egresadas de una universidad de la región del Biobío sobre su experiencia en la formación inicial. Este estudio de tipo cuantitativo utilizó una encuesta elaborada ad hoc, que se aplicó 115 docentes con egreso de la Universidad de Concepción, que analizaba tres temáticas: la primera relacionda con la satisfacción de la formación recibida; la segunda sobre las brechas con los estándares pedagógicos para la formación de profesorado; y una tercerea que tenía por objetivo caracerizar las oportundiades laborales (Soto-Hernández y Díaz Larenas, 2018). Los resultados expresan que las altos niveles de satisfacción se asocian a la formación diciplinar y teórica. Por consiguiente, las personas egresadas "evalúan de manera sobresaliente este ámbito: un $25 \%$ califica la formación disciplinar con nota 7,0 , mientras que un $43 \%$, evalúa con un 6,0"; destaca la formación disciplinar por sobre los otros ámbitos (Soto-Hernández y Díaz Larenas, 2018, p. 204). Las áreas que obtienen menores porcentajes son aquellas relacionadas con el sistema educacional chileno, políticas vigentes y la profesión docente. Finalmente el estudio identifica que "tres de cada diez encuestados considera que su formación inicial no los preparó para atender la diversidad en el aula ... [ni] conocen cómo se genera y transforma la cultura escolar" (Soto-Hernández y Díaz Larenas, 2018, p. 213). Considerando los resultados anteriores, se propone introducir estas temáticas descendidas en los procesos de innovación curricular en los programas que forman docentes. Una de las propuestas implementas por esta casa de estudios significó añadir competencias docentes en el ámbito del dominio de la comunicación oral en todas las carreras de pedagogía e intauraron un eje práctico de trabajo formativo en aspectos de evaluación y estrategias de aprendizaje por medio de un proceso de supervisión (Soto-Hernández y Díaz Larenas, 2018). 
http://doi.org/10.15359/ree.26-1.13

http://www.una.ac.cr/educare

educare@una.ac.cr

Una tercera investigación realizada por Gaete et al. (2016) tuvo por objetivo conocer las áreas que no se abordaban de manera suficiente en los programas de la FID. La metodología empleada en este estudio fue de tipo cualitativa y participaron en un grupo focal 70 docentes de enseñanza básica representantes de siete escuelas distribuidas en la zona norte, centro y sur de Chile. Los resultados del estudio indicaron la carencia del desarrollo de habilidades en el dominio de grupo tales como: la atención a la diversidad; el control disciplinar; la creación de un ambiente adecuado para el aprendizaje; y, finalmente una escasa formación en evaluación para el aprendizaje (Gaete et al., 2016).

Ferrada et al. (2013) desarrollaron una investigación cuyo énfasis era conocer las necesidades de formación curricular de profesorado en ejercicio, con más de 23 años de experiencia, en contextos de alta vulnerabilidad. La metodología incluía un diseño cualitativo de casos múltiples, en seis establecimientos educacionales públicos situados en comunas demográfica y culturalmente diversas. Los resultados indican incluir un eje de desempeños en contextos culturalmente diversos: lo que implica conocer la urbanidad marginal, la ruralidad, la indigeneidad, el trabajo en red y trabajo con las familias. Mientras que el segundo eje a trabajar implica incluir en la FID del personal docente, un diseño de una trayectoria de prácticas progresivas que sean flexibles e incorporen saberes de otras áreas que permitan desarrollar herramientas para el contexto donde se desenvuelva el estudiantado (Ferrada et al., 2013).

Una quinta investigación dirigida por Ferrada et al. (2018, citando a Ferrada et al., 2014; Ferrada, 2019), aborda la presencia de temáticas asociadas a políticas educativas en la carrera de Pedagogía en Educación básica de la región del Biobío. Para tal fin, el estudio se constituye desde el paradigma participativo y asume "la investigación dialógica-kishu kimkelay ta che (p. 263). La muestra de la investigación está compuesta por cinco casos de estudio. Los tres primeros corresponden a una universidad privada y otra pública pertenecientes al Consejo de Rectores de las Universidades Chilenas (CRUCH), y una universidad privada no perteneciente al $\mathrm{CRUCH}$. El cuarto caso corresponde a escuelas públicas de la región del Biobío con un índice de vulnerabilidad (IVE) superior al 80\%. El quinto caso corresponde a los estándares orientadores para las carreras de pedagogía en educación básica (Ferrada et al., 2018). Entre los resultados que aportan a la discusión sobre las debilidades de los programas formativos, se distingue el énfasis en el conocimiento del marco regulatorio en torno a las políticas educativas, vale decir, los estándares que regulan la formación del profesorado en esta temática descansa en cuatro competencias que se agrupan en "conocimiento del marco regulatorio nacional, una con su ajuste al trabajo escolar, y la otra refiere a la crítica de la política curricular nacional" (Ferrada et al., 2018, p. 273). Por el contrario, las competencias que demandan el profesorado en ejercicio no se restringen solo a la aplicación de normativas y conocimiento del marco regulatorio, sino que manifiestan una alta necesidad en tener incidencia en la participación en creación y discusión de las políticas educativas. En palabras del personal docente, la experiencia que les 
http://doi.org/10.15359/ree.26-1.13

http://www.una.ac.cr/educare educare@una.ac.cr

provee trabajar en dependencias municipales con altos niveles de vulnerabilidad les permite orientar y discutir, de mejor manera, la viabilidad de las políticas para el sector educacional (Ferrada et al., 2018).

Finalmente, las investigaciones lideradas por Weinstein et al. (2018) y Weinstein et al. (2019) tienen por propósito dar a conocer la opinión de los entes directivos sobre la aplicación de políticas educativas tales como: Ley de inclusión, Ley de Carrera Docente, y Ley de Nueva Educación Pública diseñadas e implementadas en el gobierno de la presidenta Michelle Bachelet en el periodo 2014 al 2017. El diseño metodológico de las investigaciones es cuantitativo, y utilizan una encuesta denominada La voz de los directores cuyo público objetivo son el personal directivo de escuelas de todo el país (Weinstein et al., 2018; Weinstein et al, 2019); así, desde el año 2013 al 2018 se aplicó, a un promedio de 585 sujetos directores de escuelas públicas y subvencionadas. Los resultados de los estudios liderados por Weinstein et al. (2018) y Weinstein et al. (2019) establecen convergencia entre la opinión directiva de las distintas dependencias, quienes concuerdan que uno de los mayores obstáculos para mejorar la calidad de la educación chilena recae en la formación inicial docente y sitúan, como urgente, que este sea la principal medida para lograrlo (Weinstein et al., 2018; Weinstein et al., 2019). Por esta razón, la ley 20.903 (MINEDUC, 2016) que creó el sistema de Desarrollo Profesional Docente -más conocida como Ley de Carrera Docente- cuenta con el apoyo de los sujetos directores, quienes ven positivamente que se instauren requisitos progresivos para el ingreso de las futuras personas docentes en las instituciones de educación superior. A su vez, les parece acertado que esta ley asegure mejoras en las remuneraciones del profesorado y resguarde tiempos de trabajo no lectivos. Un $56 \%$ los sujetos directores de establecimientos públicos y $51 \%$ de establecimientos particulares subvencionados concuerdan en sentirse con menor capacidad profesional para implementar medidas de la formación en servicio tales como: “"'implementar la inducción de docentes nuevos','atraer y contratar nuevos docentes a raíz del aumento de las horas no lectivas' y'diseñar nuevos planes de desarrollo profesional docente adecuados a las necesidades de los docentes'"' (Weinstein et al., 2019, p. 25).

\section{Estándares y resultados de la Prueba Inicia}

Como se ha mencionado en los apartados anteriores, la política que creó el Programa Inicia estaba conformada por tres componentes: Estándares; la Prueba Inicia o evaluación al final del proceso formativo y; un programa de apoyo a las IES para la innovación curricular. Algunas opiniones emanadas desde la creación de la política educativa ven, en estos mecanismos de control, una oportunidad de realizar reformas basadas en evidencia a través de datos concretos (Sotomayor y Gysling, 2011). Es decir, contar con información científica del nivel de desempeño alcanzado por el personal docente al terminar su trayectoria formativa por medio de una evaluación estandarizada, sería un mecanismo eficaz y concreto a la hora de tomar decisiones 
http://doi.org/10.15359/ree.26-1.13

http://www.una.ac.cr/educare

educare@una.ac.cr

y realizar ajustes (Joyce y Cartwright, 2018). Ahora bien, hay investigaciones que consideran que estos mecanismos son el reflejo del impacto de las políticas neoliberales heredadas del periodo de gobierno autoritario en Chile (Ávalos, 2014; Montecinos, 2014) las que, luego de cada reforma, intentan que la educación superior ingrese al proceso de rendición de cuentas, de la que el sistema escolar chileno es parte.

Sobre los diversos programas instaurados en la formación inicial de docentes, Montecinos (2014) realiza un estudio que analiza de manera crítica la implementación de los mecanismos definidos para el fortalecimiento de su formación (Prueba Inicia, estándares orientadores para la formación inicial, entre otros). En ella, desarrolla cuatro estudios diferentes con la participación de cinco universidades regionales que comprenden 13 carreras de pedagogía (Montecinos, 2014). En el caso de los dos primeros estudios tenían por objetivo conocer la percepción de los beneficios y obstáculos de los establecimientos educacionales que colaboran con la formación de profesorado. La metodología fue de carácter cuantitativa, a través del envío de una encuesta a 91 centros educativos, de los cuales 172 directivos respondieron. Los resultados indicaron que, entre los beneficios percibidos, las escuelas esperan de las universidades que la práctica estudiantil les permita ampliar el capital humano, mejorar los aprendizajes, y favorecer la actualización profesional del personal docente en ejercicio (Montecinos, 2014). Por otro lado, los obstáculos están asociados, principalmente, al rol de las universidades. Sobre estas se revela que sus estudiantes no poseen competencias pedagógicas o disciplinarias suficientes, que las tareas de la práctica son poco atingentes a la cultura escolar y que existe una escasa supervisión (Montecinos, 2014). El tercer estudio utiliza una encuesta online auto aplicada a 56 personas supervisoras de práctica de trece carreras de pedagogía. Los datos del estudio sugieren que, en general, hay una escasa disposición por parte del profesorado que recibe estudiantes en práctica por aceptar nuevas metodologías de enseñanza. El cuarto estudio distingue las percepciones del personal docente en ejercicio en su rol como mentor que recibe estudiantes en práctica. Los resultados de la investigación, señalan que el personal académico de las universidades no comprende que el diseño de una planificación que este mismo aprueba dista de las respuestas reales de estudiantes (Montecinos, 2014).

A su vez, la investigación de Rodríguez Garcés y Castillo Riquelme (2014) tuvo por objetivo describir los resultados de la Prueba Inicia en el personal docente egresado de las distintas universidades chilenas y, verificar el desempeño de las instituciones de educación superior que ofrecen carreras de pedagogía. Este estudio utilizó los resultados de la Prueba Inicia de la cohorte de 3.219 estudiantes que egresaban del año 2011. El análisis de los datos es de tipo descriptivo. Sobre los resultados de la Prueba Inicia, las investigaciones revisadas plantean que "alrededor del 60\% del profesorado evaluado en la Prueba Inicia 2011 carecía de las competencias básicas para ejercer la labor docente en el sistema escolar chileno" (Rodríguez Garcés y Castillo Riquelme, 2014, p. 11). Los resultados también indican que entre los resultados de la prueba Inicia y los años 
http://doi.org/10.15359/ree.26-1.13

de acreditación de las carreras de pedagogía "se encuentran moderadas correlaciones, cuyos índices, siendo significativos, no superan un rho de 0.3 para cada una de las pruebas y en la totalidad del sistema de Educación Superior" (Rodríguez Garcés y Castillo Riquelme, 2014, p. 17).

Por otra parte, Domínguez et al. (2012) realizaron una investigación que tenía por objetivo conocer la coherencia entre la acreditación implementada por la CNA y la Prueba Inicia entre los años 2009 y 2010 para las carreras de pedagogía básica. La metodología utilizada en este estudio contempló el análisis de los puntajes de las cohortes 2009 y 2010 de estudiantes que rindieron la Prueba Inicia y los resultados de la acreditación realizada por las agencias acreditadoras (Domínguez et al., 2012). Los resultados reflejan que existe una correlación positiva pero baja entre "INICIA y los años de acreditación, que van entre 0,12 y 0,23" (Domínguez et al., 2012, p. 23) y asume que el puntaje de ingreso de estudiantes en la PSU pronostica más cercanamente el logro de estudiantes en la Prueba Inicia que los años de acreditación de la carrera (Domínguez et al., 2012; Rufinelli, 2013).

Finalmente, la investigación de Pedraja-Rejas et al. (2012) pretendió conocer el valor agregado de las instituciones de educación superior en la formación de profesorado. Para ello, se utilizó una metodología cuantitativa que analizó los resultados obtenidos en la Prueba Inicia del año 2010 y la relación con los años de acreditación de la CNA. Pedraja-Rejas et al. (2012) afirman que existe relación entre el puntaje promedio de la Prueba Inicia obtenido por las personas egresadas de pedagogía, y los años de acreditación de las universidades. En consecuencia "los años de acreditación explican en un 56,7\% los resultados de la Prueba Inicia" (Pedraja-Rejas et al., 2012, p. 22), lo que podría explicar que los años de acreditación de la institución podrían influir positivamente en la formación de docentes.

\section{Discusiones}

A partir de los resultados obtenidos tras los análisis, se identificaron cuatro líneas que fueron reconocidas por los estudios como políticas claves: el fortalecimiento a la formación inicial docente, la creación del Programa de Mejoramiento de la Calidad y Equidad en la Educación Superior (Mecesup), la creación del Consejo Nacional de Acreditación de Pregrado (Actual CNA) y el programa Inicia. Al respecto, podemos establecer que las políticas emanadas desde el Estado para el mejoramiento en la formación de profesorado han cumplido con la etapa de implementación en las universidades. No obstante, aún existen nudos críticos en esta formación local y global, como, por ejemplo, en la formación del profesorado europeo (García, 2007). Al respecto, un eje necesario de revisar es el divorcio entre la formación práctica y la teoría, que permita orientar la formación docente incorporando perspectivas que preparen al futuro profesorado para generar conocimiento sobre la enseñanza y contribuir a la sociedad del conocimiento, propiciando diálogos entre los entes formadores universitarios y los profesorados guías o personal mentor de los establecimientos educativos. 
http://doi.org/10.15359/ree.26-1.13

http://www.una.ac.cr/educare

educare@una.ac.cr

El segundo tema que se desprende de la revisión alude al tipo de estudiantado que ingresa a estudiar pedagogía. Así, quienes ingresan a carreras de educación poseen puntajes mínimos establecidos para el ingreso y, provienen de los dos quintiles de menores ingresos de la población chilena (Ávalos, 2004; Inzunza et al., 2011). Esta situación genera a priori un gran desafío para las IES porque deben entregar una formación a estudiantes que ya poseen brechas significativas respecto a los otros grupos profesionales. A su vez, las reflexiones teóricas dejan entrever un efecto no deseado de estas políticas. El primer efecto es el descenso en la matrícula en universidades selectivas ya que, estudiantes que no logran los puntajes mínimos exigidos, optan por matricularse en universidades con escasa o nula selectividad. Esta acción incrementa las brechas de calidad en la formación del profesorado que egresa de instituciones de educación superior selectivas versus aquellas universidades con escasa o nula selectividad (Inzunza et al., 2011).

Una tercera temática señala la escasa relación entre la formación recibida por el futuro profesorado en la universidad y el quehacer durante su formación de la práctica en los establecimientos educacionales. Los resultados de las investigaciones apuntan que esta articulación está pendiente. Sin embargo, se han impulsado normativas en los nuevos estándares de acreditación para las carreras de pedagogía y la Ley de Desarrollo Profesional Docente (Ley 20.903), que insisten en el compromiso de direccionalidad entre las escuelas y universidades que forman docentes.

Otro de los temas que se releva en la presente revisión, es la debilidad que percibió el egresado de las carreras de pedagogía en torno a su formación con el ámbito pedagógico. Señalaron que el trabajo con la familia, el manejo conductual, la diversidad, la cultura escolar y la evaluación fueron los elementos ausentes o poco tratados durante su formación universitaria.

Un elemento transversal que emergió tras los análisis fue la percepción compartida entre docentes en ejercicio y estudiantes que egresan de las carreras de pedagogía, quienes reconocen que aun cuando las mallas formativas incorporan y actualizan conocimiento sobre las adecuaciones del currículo oficial, la normativa vigente y la política educativa chilena, existen escasos espacios de acceso para la discusión y contribución a dichas normativas y políticas educativas. Así, el conocimiento situado que los docentes en servicio y recién egresados construyen desde su ejercicio profesional, no es incorporado a dichas discusiones.

\section{Conclusiones}

Los resultados de este estudio permitieron identificar las políticas que han marcado el mejoramiento a la formación de profesorado en Chile, y que fueron implementadas en las instituciones de educación superior que forman docentes. Además, estas políticas permitieron mejorar las desregulaciones que sufrieron las carreras de pedagogía durante el periodo de 
http://doi.org/10.15359/ree.26-1.13

la dictadura militar. A consecuencia de la implementación de las políticas, las universidades formadoras de profesorado pudieron fortalecer los claustros académicos, su infraestructura y diseñar nuevos perfiles de egreso en sintonía con las políticas internacionales como las sugeridas por el Proyecto Tuning.

Por otro lado, las políticas tendientes a incentivar el acceso a las carreras de pedagogía, introdujeron mecanismos de incentivos para la atracción de estudiantes a estas carreras. Las becas fueron uno de los componentes que tuvieron mayor éxito en un contexto donde la educación superior en Chile era costosa. Esta política logró su objetivo, pero con el transcurso de los años, el interés por acceder a las carreras de pedagogía fue decreciendo. Como consecuencia de ello, y pese a los incentivos dispuestos para atraer estudiantes a las carreras de pedagogía, desde el año 2005 al 2020, las matrículas de las carreras de pedagogía han descendido (Consejo Nacional de Educación [CNED], 2021). Cabe preguntarse, entonces, si las becas o los beneficios como la gratuidad para estudiar carreras de pedagogía, son los mecanismos para incentivar que más estudiantes opten por ingresar a estas carreras. La evidencia señala que por sí solas, este mecanismos de gratuidad no son las más idóneas, sino que se requieren de otros elementos que hagan que esta profesión sea más atractiva.

Respecto a la débil articulación existente entre la formación de docentes, y el sistema escolar chileno, organismos supranacionales han referido recomendaciones en torno a esta temática (Organización para la Cooperación y el Desarrollo Económicos [OCDE], 2004). Ahora bien, con la implementación del programa de Fortalecimiento a la Formación Inicial de Profesores revisados en este estudio, se comenzaron a introducir, de manera gradual, la experiencia práctica en la trayectoria formativa del futuro profesorado, la que tras la implementación de la política llegó a un promedio cercano al 20\% (Montecinos et al., 2010). A pesar de los avances, esta temática tiene poca evidencia en Chile, y existen dudas sobre cómo se ha desarrollado este proceso. Ahora bien, se constata que existe una implementación desde las universidades en el diseño de la trayectoria formativa que incluye un porcentaje mayor de experiencias tempranas, intermedias y profesionales de la formación práctica en los establecimientos educacionales, pero no del proceso bidireccional entre estas dos instituciones.

De los resultados que establecen grados de relación entre las variables como Inicia, PSU o años de acreditación, llama la atención la débil correlación existente entre el resultado de la CNA y los puntajes obtenidos por estudiantes en la Prueba Inicia. Al respecto, la discusión indica que el puntaje de ingreso es el mecanismo que predice con mayor certeza el logro de los desempeños en la Prueba Inicia. Estos resultados nos llevan a reflexionar en torno a la pertinencia del tipo de evaluación que se aplica para medir los desempeños en el futuro profesorado y a los criterios utilizados por la CNA. Frente a esta situación, pareciera ser pertinente observar los instrumentos de tipo cualitativo que utilizan los países europeos, o norteamericanos para el ingreso a la docencia. Allí, predominan entrevistas semiestructuradas, intenciones de 
http://doi.org/10.15359/ree.26-1.13

http://www.una.ac.cr/educare

educare@una.ac.cr

investigación, y desarrollo de habilidades pedagógicas transversales a través de la escritura y simulación de clases (Pérez Granados, 2015). Cabe señalar que la Prueba Inicia terminó de aplicarse el año 2015, y desde el año 2016 se aplica la Evaluación Nacional Diagnostica (END). La END ha evaluado tres cohortes de estudiantes. Dado el contexto reciente de esta evaluación, aún no existen investigaciones científicas sobre este proceso, y solo contamos con información entregada por informes gubernamentales que señalan que $91,1 \%$ del estudiantado matriculado en el penúltimo año de la carrera rindió la evaluación el 2018 y que respecto a la evaluación anterior -2017- los puntajes subieron un 38\% (CPEIP, 2019).

De esta manera, los análisis de esta revisión con base en las investigaciones indican que aún persisten debilidades en los programas que forman docentes. En general, los estudios coinciden en relevar los conocimientos y saberes emanados desde el ejercicio profesional como un insumo valioso a considerar para la formación inicial. Entre las sugerencias, el profesorado en ejercicio indica que los procesos de innovación curricular que realizan las facultades de educación deberían contemplar saberes pedagógicos como: el trabajo en diversidad, el desarrollo de habilidades reflexivas que ayuden al futuro profesorado a flexibilizar el quehacer profesional en las aulas chilenas y la evaluación para el aprendizaje (Ferrada et al., 2013).

Finalmente, entre las limitantes de este artículo, cabe señalar que esta revisión es un estudio exploratorio que describió los hitos más importantes que han marcado la formación inicial de profesorado en Chile, con base en las investigaciones disponibles. Como proyección para nuevas investigaciones se recomienda ampliar la búsqueda integrando artículos que aborden la política pública de gratuidad en educación superior en Chile, y precisar en los criterios de búsqueda los años de creación de las políticas en los años noventa, de manera de marcar un periodo histórico en el que fueron elaboradas. También y considerando el actual contexto sanitario que se enfrentó, se requiere incorporar criterios que aludan al uso de tecnologías educativas en el proceso de formación de docentes en Chile.

\section{Declaración de Material complementario}

Este artículo tiene disponible, como material complementario:

-La versión preprint del artículo en https://doi.org/10.5281/zenodo.4891086 
http://doi.org/10.15359/ree.26-1.13

http://www.una.ac.cr/educare educare@una.ac.cr

\section{Referencias}

Alvarado, J., Arellano, J. P., Aylwin, M., Beyer, H., Brunner, J. J., Krebs, A., Matte, P., Molina, S., Pavez, J., Romaguera, P., Rosso, P. P. y Zalaquett, P. (2010). Informe final: Primera etapa. Propuestas para fortalecer la profesión docente en el sistema escolar chileno. https://docplayer.es/8309536Informe-final-primera-etapa-propuestas-para-fortalecer-la-profesion-docente-en-elsistema-escolar-chileno.html

Ávalos, B. (2004). La formación docente inicial en Chile. http://www.ub.edu/obipd/PDF\%20docs/ Aspectes\%20laborals/Documents/La\%20Formacion\%20Docente\%20Inicial\%20en\%20 Chile.\%20AVALOS.pdf

Ávalos, B. (2014). La formación inicial docente en Chile: Tensiones entre políticas de apoyo y control. Estudios Pedagógicos, 40(Especial), 11-28. https://doi.org/10.4067/S0718$\underline{07052014000200002}$

Bellei, C. (2010). El Talón de Aquiles de la reforma. Análisis sociológico de la política de los 90 hacia los docentes en Chile. En S. Martinic y M. Pardo (Eds.), Economía política de las reformas educativas en América Latina (pp. 130-287). CIDE; PREAL. https://repositorio.uahurtado.cl/ $\underline{\text { handle/11242/8409 }}$

Castro Rubilar, J. I. (2017). La formación inicial docente en Chile: Una parte de su historia y los desafíos de aprendizaje por competencias. Praxis Educativa, 21(2), 12-21. https://doi. org/10.19137/praxiseducativa-2017-210202

Centro de Perfeccionamiento, Experimentación e Investigación Pedagógica. (2019). Informe resultados nacionales. Evaluación nacional diagnóstica de la formación inicial docente 2019. https://www.cpeip.cl/wp-content/uploads/2020/08/Informe-Nacional-END-2019 rect.pdf

Consejo Nacional de Educación. (2021). Matrícula Instituciones/Programas Educación Superior. https://www.cned.cl/indices/matricula-institucionesprogramas-educacion-superior

Contreras-Sanzana, G., y Villalobos-Clavería, A. (2010). La formación de profesores en Chile: Una mirada a la profesionalización docente. Educación y Educadores, 13(3), 397-417. https:// doi.org/10.5294/edu.2010.13.3.5

Cox, C., y Gysling, J. (2009). La formación del profesorado en Chile 1842-1987. CIDE.

Cox, C., Meckes, L. y Bascopé, M. (2010). La institucionalidad formadora de profesores en chile en la década del 2000: Velocidad del mercado y parsimonia de las políticas. Revista Pensamiento Educativo, 46(1), 205-245. https://pensamientoeducativo.uc.cl/files/ journals/2/articles/468/public/468-1034-1-PB.pdf 
http://doi.org/10.15359/ree.26-1.13

http://www.una.ac.cr/educare

educare@una.ac.cr

Domínguez, M., Bascopé, M., Meckes, L. y San Martín, E. (2012). ¿Producen mejores resultados las carreras de pedagogía básica con más años de acreditación? Estudios Públicos, 128(Primavera), 1-59. https://doi.org/10.38178/cep.vi128.293

Fardella, C., y Sisto, V. (2015). Nuevas regulaciones del trabajo docente en Chile: Discurso, subjetividad y resistencia. Psicologia \& Sociedade, 27(01), 68-79. https://doi. org/10.1590/1807-03102015v27n1p068

Ferrada, D., Turra, O. y Villena, A. (2013). Currículum transformador de formación inicial para profesores en contextos de vulnerabilidad social. Cadernos de Pesquisa, 43(149), 642-661. https://doi.org/10.1590/S0100-15742013000200013

Ferrada, D., Villena, A., y del Pino, M. (2018). ¿ Hay que formar a los docentes en políticas educativas? Cadernos de Pesquisa, 48(167), 254-279. https://doi.org/10.1590/198053144740

Gaete,A., Gómez,V.y Bascopé,M.(2016).¿Quéle piden los profesores a la formación inicial docente en Chile? Temas de la Agenda Pública, 11(86), 1-18. https://politicaspublicas.uc.cl/wpcontent/uploads/2016/06/Qu\%C3\%A9-le-piden-los-profesores-a-la-formaci\%C3\%B3ninicial-docente-en-Chile.pdf

García, C. M. (2007). La formación docente en la sociedad del conocimiento y la información: Avances y temas pendientes. Olhar de Professor, 10(1),63-90. https://www.redalyc.org/ articulo.oa?id=68410104

Inzunza, J., Assaél, J. y Scherping, G. (2011). Formación docente inicial y en servicio en Chile: Tensiones de un modelo neoliberal. Revista Mexicana de Investigación Educativa, 16(48), 267-292. https://www.researchgate.net/publication/262783409_Formacion docente inicial y en servicio en Chile tensiones de un modelo neoliberal

Joyce, K. E. y Cartwright, N. (2018). Meeting our standards for educational justice: Doing our best with the evidence. Theory and Research in Education, 16(1), 3-22. https://doi. org/10.1177/1477878518756565

Ministerio de Educación. (1 de abril, 2016). Ley N.o 20903. Crea el sistema de desarrollo profesional docente y modifica otras normas. Diario Oficial de la República de Chile, Núm 41.421.- Año 139 - N.8815.596 (M. R.), pp. 1-24. https://www.diariooficial.interior.gob.cl/ media/2016/04/01/do-20160401.pdf

Ministerio de Educación. (2019). Programa MECESUP. Mejoramiento de la calidad y la equidad en la educaciónterciaria.http://dfi.mineduc.cl/index2.php?id portal=59\&id seccion=3586\&id contenido $=14892$ 
http://doi.org/10.15359/ree.26-1.13

http://www.una.ac.cr/educare educare@una.ac.cr

Montecinos, C., Walker, H., Solís, M. C., Núñez, C., Contreras, I. y Rittershaussen, S. (2010). 9. Lineamientos para el diseño del currículo del área de formación de carreras de carreras de pedagogía. En S. Martinic y G. Elacqua (Eds.), ¿Fin de ciclo? Cambios en la gobernanza del sistema educativo (pp. 233-255). Unesco; Universidad Católica de Chile. https://docplayer. es/13955397-Fin-de-ciclo-cambios-en-la-gobernanza-del-sistema-educativo-editoressergio-martinic-gregory-elacqua.html

Montecinos, C. (2014). Análisis crítico de las medidas de presión propuestas para mejorar la formación inicial de docentes en Chile por el panel de expertos para una educación de calidad. Estudios Pedagogicos, 40(Especial), 285-301. https://doi.org/10.4067/S0718$\underline{07052014000200017}$

Núñez Prieto, I. (2007). La profesión docente en Chile: Saberes e identidades en su historia. Pensamiento Educativo, 41(1), 149-164. http://ojs.uc.cl/index.php/pel/article/view/25689

Organización para la Cooperación y el Desarrollo Económicos. (2010). Política de educación y formación: Los docentes son importantes. Atraer, formar y conservar a los docentes eficientes. Autor. https://doi.org/10.1787/9789264046276-es

Organización para la Cooperación y el Desarrollo Económicos. (2004). Revisión de Políticas Nacionales de Educación. Chile. Autor. https://www.oecd-ilibrary.org/education/revisionde-politicas-nacionales-de-educacion-chile 9789264021020-es

Oliva, M. A. (2010). Política educativa chilena 1965-2009. ¿Qué oculta esa trama? Revista Brasileira de Educação, 15(44), 311-410. https://doi.org/10.1590/S1413-24782010000200008

Panel de Expertos para una Educación de Calidad. (2010). Informe Final: Primera Etapa. Propuestas para fortalecer la profesión docente en el sistema escolar chileno. http://200.6.99.248/ bru487cl/files/Informe_Finaldef.pdf

Pavié Nova, A. (2012). Caracterización de la formación inicial docente en Chile. Revista Lider, 14(20), 199-219. http://ceder.ulagos.cl/lider/images/numeros/20/[LIDERVol20A\%C3\%B10142012-ISSN-0717-0165]8.-CaracterizaciondelaformacioninicialdocenteenChile.pdf

Pedraja-Rejas, L.M.,Araneda-Guirriman,C.A., Rodríguez-Ponce, E. R.y Rodríguez-Ponce,J.J.(2012). Calidad en la formación inicial docente: Evidencia empírica en las universidades chilenas. Formacion Universitaria, 5(4), 15-26. https://doi.org/10.4067/S0718-50062012000400003

Pérez Granados, L. (2015). Análisis de las pruebas de acceso a la formación de docentes en España y Finlandia: Conocimientos o competencias. Revista Complutense de Educacion, 26(3), 591-609. https://doi.org/10.5209/rev RCED.2015.v26.n3.44448 
http://doi.org/10.15359/ree.26-1.13

http://www.una.ac.cr/educare

educare@una.ac.cr

Reich, R. (2009). Programa Mecesup2. Diez años de implementación. En Consejo Superior de Educación (Autor), Seminario Internacional 2008. Políticas de Educación Superior: Explorando Horizontes, Riesgos y Posibilidades (pp. 127-133). Autor. https://www.cned. $\mathrm{cl} /$ seminario/politicas-de-educacion-superior-explorando-horizontes-riesgos-yposibilidades-ano-2008

Rodríguez Garcés, C. y Castillo Riquelme, V. (2014). Calidad en la formación inicial docente: Los déficits en las competencias pedagógicas y disciplinares en Chile. Actualidades Investigativas en Educación, 14(2), 1-25. https://doi.org/10.15517/aie.v14i2.14812

Sánchez, G. (2014). Los estándares pedagógicos en el desempeño de estudiantes de pedagogía básica en procesos de práctica profesional, 2012: Un diálogo a varias voces. Revista Investigaciones en Educación, 14(1), 69-89. http://revistas.ufro.cl/ojs/index.php/educacion/ issue/view/112

Ruffinelli, A. (2013). La calidad de la formación inicial docente en Chile: La perspectiva de los profesores principiantes. Calidad en la Educación, (39), 118-154. https://doi.org/10.31619/ caledu.n39.80

Soto-Hernández, V. y Díaz Larenas, C. H. (2018). Formación inicial docente en una universidad chilena: Percepciones de sus egresados. Praxis \& Saber, 9(20), 191-216. https://doi. org/10.19053/22160159.v9.n20.2018.8429

Sotomayor, C. y Gysling, J. (2011). Estándares y regulación de calidad de la formación de profesores: Discusión del caso chileno desde una perspectiva comparada. Calidad en la Educación, (35), 91-129. https://doi.org/10.31619/caledu.n35.97

Vaillant, D. (2018). Estudio exploratorio sobre los modelos organizacionales y pedagógicos de instituciones dedicadas a la formación docente inicial. Un análisis en clave comparada. Informe final. INFOD e IIPE-UNESCO. https://panorama.oei.org.ar/ dev2/wp-content/ uploads/2018/05/Informe-final-Estudio-formaci\%C3\%B3n-docente-INFOD.pdf

Weinstein, J., Muñoz, G. y Rivero, R. (2018). Los directivos escolares como informantes cualificados de las políticas educativas. Sus opiniones bajo el gobierno de Michelle Bachelet en Chile (2014-2017). REICE. Revista Iberoamericana Sobre Calidad, Eficacia y Cambio en Educación, 16(3), 5-27. https://doi.org/10.15366/reice2018.16.3.001

Weinstein, J., Muñoz, G., Sembler, M., y Rivero, R. (2019). La opinión de directores de centros públicos y privados sobre las políticas educativas de Chile. Profesorado, Revista de Currículum y Formación del Profesorado, 23(2), 13-39.https://doi.org/10.30827/profesorado. v23i2.9285 